\title{
Wirkt eine Kombination von Dexpanthenol und Glycerin stärker hydratisierend als die Einzelkomponenten allein?
}

\section{Gloor Bettina Senger W. Gehring}

\author{
Do Dexpanthenol/Glycerin Combinations Achieve Better Skin Hydration \\ than Either Component Alone?
}

\section{Zusammenfassung}

Zielsetzung: Werden Moisturizer kombiniert, so kommt es nicht immer zu einer additiven Wirkung. Überprüft werden soll, ob Dexpanthenol und Glycerin sich in ihrer Wirkung in einem Präparat addieren, das nach Bad und Sonnenexposition angewendet wird. Methodik: Bei 20 Versuchspersonen wurde eine standardisierte Lichtbestrahlung vorgenommen. Anschließend wurden Zubereitungen vom O/W Typ mit 2\% Dexpanthenol bzw. 3\% Glycerin oder mit der Kombination dieser Wirkstoffe zur Anwendung gebracht. Gemessen wurde bei Versuchsbeginn sowie nach 24 und 72 Stunden die Hornschichtfeuchtigkeit mit dem Corneometer. Ergebnisse: Die Lichtexposition selbst lässt die Hornschichtfeuchtigkeit unbeeinflusst. Beide Wirkstoffe führen zu einer Hydratisierung. In der Kombination addiert sich dieser Effekt. Schlussfolgerung: Kombinationen von Dexpanthenol und Glycerin sind bezüglich der hydratisierenden Wirkung sinnvoll, da man die Glycerinkonzentration im Hinblick auf die wegen des Klebeeffektes eingeschränkte kosmetische Akzeptanz gerne relativ niedrig hält.

\section{Abstract}

Objective: The combination of different moisturisers does not always lead to an additive effect. The aim of this study is to investigate whether the effects of dexpanthenol and glycerin combined in a single product to be used after bathing and exposure to the sun are additive. Method: 20 subjects were subjected to standardised light radiation. Oil-in-water preparations containing $2 \%$ dexpanthenol, $3 \%$ glycerin or their combination were then applied to the skin. The hydration of the horny layer was subsequently measured with a corneometer at beginning, after 24, and after 72 hours. Results: Exposure to light itself has no effect on the moisture content of the stratum corneum. Both active ingredients were found to moisturise the skin and to have an additive effect when combined. Conclusion: Combinations of dexpanthenol and glycerin are useful as moisturisers as the glycerin content can be kept relatively low in view of the reduced cosmetic acceptance resulting from its stickiness.

\section{Einleitung}

Wasserkontakte und möglicherweise zusätzlich die UV-Bestrahlung vermindern die Hornschichtfeuchtigkeit. Dexpanthenol weist einen hydratisierenden Effekt auf die Hornschicht im einfachen Anwendungstest und im Natriumlaurylsulfatirritationstest auf $[1,2]$. Ein hydratisierender Effekt ist außerdem von Glycerin bekannt $[3,4]$. Überprüft werden soll, ob Glycerin und Dexpanthenol allein und in Kombination die Hornschichtfeuchtigkeit bei Applikation nach einer Lichtexposition steigern.

\section{Material und Methodik}

\section{Prüfpräparate}

Das Prüfpräparat selbst ist die Entwicklung Daylong après der Firma Spirig Pharma AG, Egerkingen, Schweiz, mit einem Zusatz von Glycerin und Dexpanthenol (PP). Es handelt sich dabei um eine $\mathrm{O} / \mathrm{W}$ Emulsion mit folgender Zusammensetzung nach INCI:

- Dexpanthenol 2\%

- Glycerin 3\%

- Aqua 


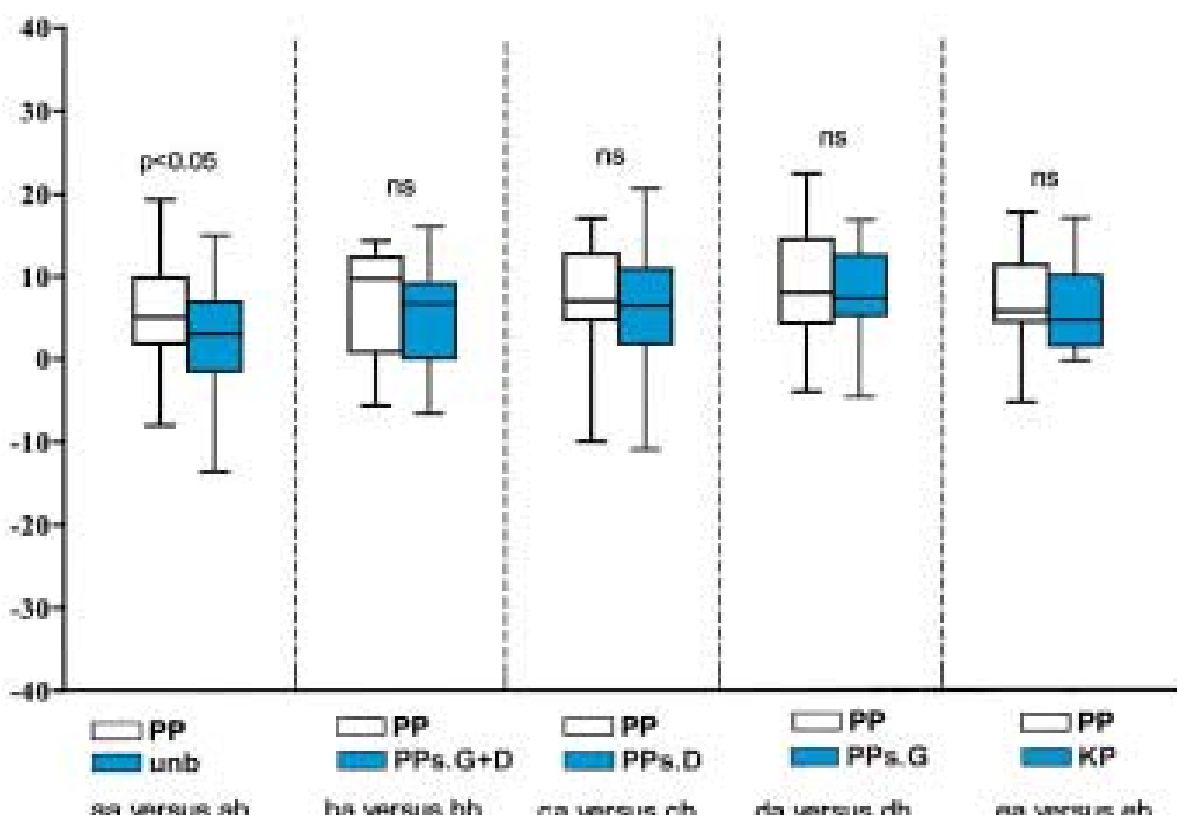

aa versus ab baversus bb ca versus cb daversus db a versus ab
Abb.1 Messwertdifferenzen zwischen den Messungen nach 24 Stunden und den Ausgangswerten. Angegeben sind die Mediane, die Boxen ( 25 und $75 \%$ Perzentile) sowie die Maxima und Minima. Nebeneinander gestellt sind die Messwertdifferenzen zwischen aa und $a b$, ba und bb, ca und cb, da und db bzw. ea und eb. Die statistischen Angaben beziehen sich jeweils auf die Seitenvergleiche aa/ab, ba/bb, ca/cb, da/db und ea/eb. Bezüglich aa/ab und ea/eb erfolgte die Prüfung zweiseitig, bezüglich ba/bb, ca/ $\mathrm{cb}$ und $\mathrm{da} / \mathrm{db}$ einseitig.

PP: Prüfpräparat; unb: unbehandelt; PP s. $G+D$ : Prüfpräparat ohne Glycerin und Dexpanthenol; PP s. D: Prüfpräparat ohne Dexpanthenol; PP s. G: Prüfpräparat ohne Glycerin; KP: Konkurrenzprodukt.

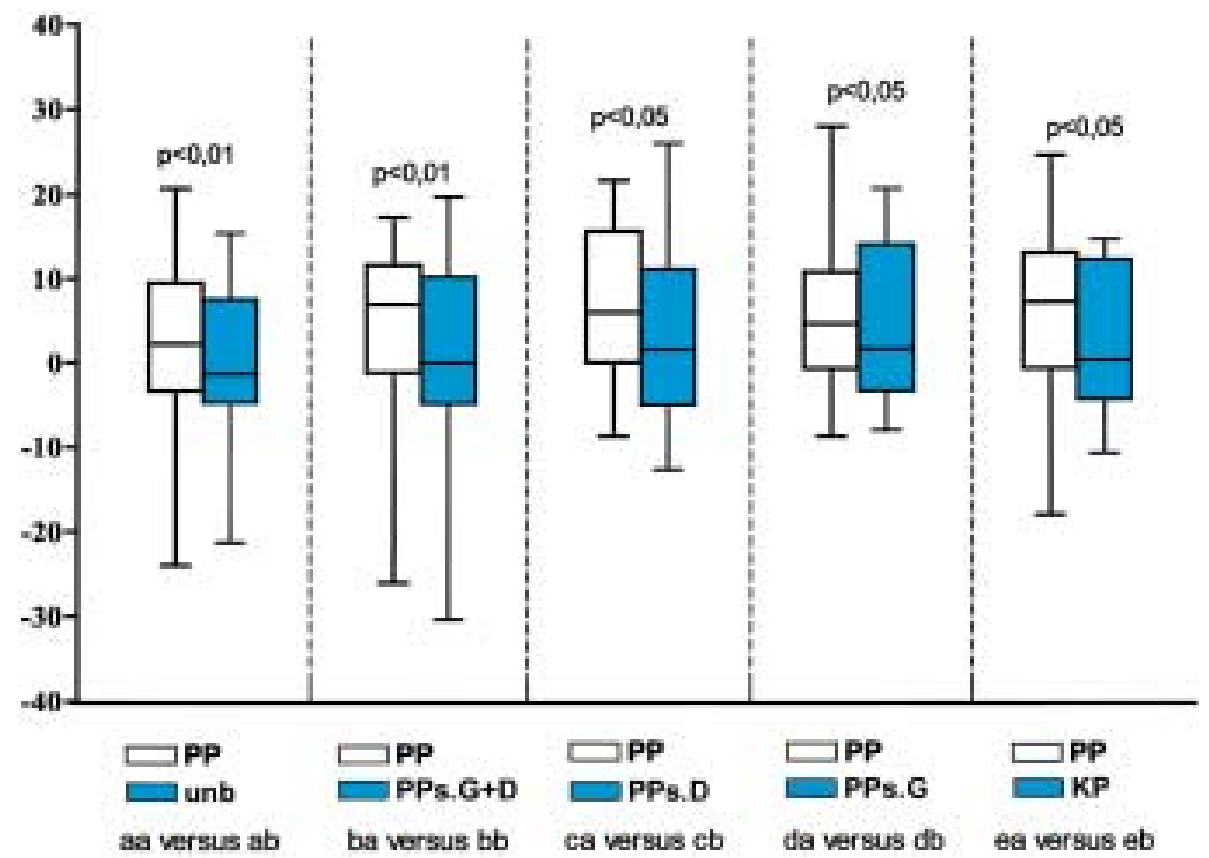

Abb. 2 Messwertdifferenzen zwischen den Messungen nach 72 Stunden und den Ausgangswerten. Angegeben sind die Mediane, die Boxen ( 25 und 75\% Perzentile) sowie die Maxima und Minima. Nebeneinander gestellt sind die Messwertdifferenzen zwischen aa und $a b$, ba und $b b$, ca und $c b$, da und $\mathrm{db}$ bzw. ea und eb. Die statistischen Angaben beziehen sich jeweils auf die Seitenvergleiche $a a / a b, b a / b b, c a / c b, d a / d b$ und ea/eb. Bezüglich aa/ab und ea/eb erfolgte die Prüfung zweiseitig, bezüglich ba/bb, ca/ $\mathrm{cb}$ und $\mathrm{da} / \mathrm{db}$ einseitig.

PP: Prüfpräparat; unb: unbehandelt; PP s. $G+D$ : Prüfpräparat ohne Glycerin und Dexpanthenol; PP s. D: Prüfpräparat ohne Dexpanthenol; PP s. G: Prüfpräparat ohne Glycerin; KP: Konkurrenzprodukt.
- Alcohol

- Carthamus Tinctorius

- Dimethicon

- Tocopherol

- Phosphatidylcholin

- Acrylate C10 - 30 Alkyl Acrylat Crosspolymer

- Na Hydroxid

- Carbomer

- Parfüm

Neben der Prüfzubereitung wurden identische Zubereitungen ohne Glycerin ( PP sine G ), ohne Dexpanthenol (PP sine D) sowie ohne Glycerin und Dexpanthenol (PP sine G und D) geprüft. Die- se Zubereitungen wurden von der Fa. Spirig Pharma AG in Egerkingen hergestellt. Außerdem wurde im Vergleich ein Konkurrenzpräparat (KP) untersucht.

\section{Versuchsstellen}

Als Versuchsstellen dienen in Höhe der BWS 5 übereinander liegende Stellen auf der rechten Seite und die dazu symmetrischen Versuchsstellen auf der linken Seite. Die oberste horizontale Reihe ist mit aa links und ab rechts gekennzeichnet. Die Kennzeichnung der folgenden drei horizontalen Reihen ist entsprechend ba und bb, ca und cb, da und db sowie ea und eb. Gegenübergestellt werden an den verschiedenen Prüfstellen:

a) PP (aa) versus unbehandelt ( $a b$ ) 
b) PP (ba) versus PP sine G und D (bb)

c) $P P(\mathrm{ca})$ versus $P P$ sine $G(\mathrm{cb})$

d) $P P(d a)$ versus $P P$ sine $D(d b)$

e) $P P(e a)$ versus $K P(e b)$

Die Größe der Versuchsfelder ist durch die mit dem Gerät bestrahlte Fläche (Durchmesser ca. 1,5 cm) vorgegeben. Der Abstand zwischen den Versuchsfeldern in vertikaler Richtung ist $1,5 \mathrm{~cm}$, in horizontaler Richtung $8 \mathrm{~cm}$.

Die Versuchsstellen wurden so randomisiert, dass bei jeder verglichenen Gruppe die Hälfte der Versuchspersonen auf der rechten Seite und die andere Hälfte auf der linken Seite mit PP behandelt wurde. Die dazu symmetrische Stelle wurde jeweils mit ab, $\mathrm{bb}, \mathrm{cb}, \mathrm{db}$ und eb behandelt. Die gepaarten Versuchsstellen aa/ $\mathrm{ab}, \mathrm{ba} / \mathrm{bb}, \mathrm{ca} / \mathrm{cb}, \mathrm{da} / \mathrm{db}$ und ea/eb wurden von Fall zu Fall randomisiert. Die Testprodukte ba, bb, ca, cb, da, db, ea und eb wurden durch die Firma Spirig Pharma AG verblendet. Die Studie war damit mit Ausnahme der unbehandelten Stelle und der symmetrischen Versuchsstelle doppelblind.

\section{Versuchspersonen}

Untersucht werden 20 gesunde Testpersonen, Ausschlusskriterien waren Alter unter 18 Jahre, Schwangerschaft, Stillzeit und Vorbehandlung an den Versuchsstellen innerhalb der letzten 4 Tage vor dem Versuch. Die Ethikkommission der Universität Freiburg hat für die Untersuchungen ein befürwortendes Votum abgegeben.

\section{Testvorgehen}

Vier Tage vor Beginn der eigentlichen Bestrahlung wurde eine Lichttreppe mit dem UV-Multitester der Firma Saalmann (Einstellung UVB) im Bereich der LWS untersucht, um die individuelle Strahlendosis zu ermitteln. Die applizierten Lichtenergien richteten sich nach den Angaben der Herstellerfirma und den jeweiligen Hauttypen (Typ 1-6 nach Fitzpatrick). Die Lichttreppe wurde nach $24 \mathrm{~h}$ abgelesen. Mit der niedrigsten Lichtmenge, bei der es zu einem deutlichen Lichterythem kommt (Zunahme des $a^{*}$-Wertes um mehr als 50\% und deutlich klinisch sichtbares Erythem), wurde an allen oben angegebenen Versuchsstellen ebenfalls mit dem Saalmann-UV-Tester ein Lichterythem erzeugt (Tag 1). Anschließend an die Bestrahlung wurden die Testprodukte aufgetragen. Die Auftragungsmenge war ca. $0,01 \mathrm{ml} / \mathrm{cm}^{2}$. Diese Behandlung erfolgt an drei Tagen morgens $9 \mathrm{~h}$ (an Tag 1 unmittelbar nach der Bestrahlung). Vor der Bestrahlung (Tag 1), nach 24 (Tag 2, vor der Behandlung) sowie 72 Stunden (Tag 4) erfolgten die Messungen wie unten angegeben.

\section{Messmethoden}

Die Hornschichtfeuchtigkeit wurde mit dem Corneometer CM 820 (Courage \& Khazaka, Köln) gemessen. Der Messkopf des Gerätes besteht aus zwei konzentrisch angeordneten Metallringen unterschiedlicher Größe, die durch eine Isolation voneinander getrennt wird. Diese Messsonde wird durch eine Schutzfolie vor dem direkten Kontakt mit der Haut geschützt. Messtechnisch erfasst wird das elektrische Feld in der Haut, d.h. der kapazitive Widerstand der Haut. Wegen der hohen Dielektrizitätskonstanten des Wassers ist der kapazitive Widerstand stark vom Wassergehalt abhängig. Die gültigen Guidelines wurden beachtet [5].

\section{Statistische Auswertung}

Der Auswertung zugrundegelegt werden immer die Messwertdifferenzen zwischen Tag 1 vor Versuchsbeginn einerseits und den jeweiligen nachfolgenden Messungen anderseits. Gegenübergestellt werden jeweils die symmetrischen Messstellen. Der Vergleich zwischen diesen erfolgt über den Wilcoxon-Test für Paardifferenzen. Bezüglich der Wirkung von Glycerin, Dexpanthenol und der Kombination aus Glycerin und Dexpanthenol erfolgte die Prüfung einseitig, da eine hydratisierende Wirkung beider Wirkstoffe bekannt ist. Bezüglich des Vergleiches PP versus unbehandelt und PP versus KP erfolgte die Prüfung zweiseitig.

\section{Ergebnisse}

Abb. 1 und 2 zeigen die Mediane, Boxen (25- bis 75\%-Perzentile), Minima und Maxima zu den Messzeitpunkten Tag 2 und 4. Zum Messzeitpunkt Tag 2 war nur PP signifikant unterschiedlich im Vergleich zu unbehandelt. Zum Zeitpunkt Tag 4 unterschied sich PP signifikant von Unbehandelt, KP, PP sine G und D, PP sine D und PP sine G. Die Kombination von Dexpanthenol und Glycerin führt also zu einer besseren Hydratisierung als das Vehikel sowie als Glycerin allein und Dexpanthenol allein.

\section{Diskussion}

Gehring et al. haben eine hydratisierende Wirkung von 2,5\% Dexpanthenol im einfachen Anwendungstest [1] und im NLS-Irritationstest [2] nachweisen können. Die Unterschiede waren teilweise signifikant. Es bestanden deutliche Unterschiede in Abhängigkeit vom verwendeten Vehikel. Vielfältig nachgewiesen wurde eine hydratisierende Wirkung von Glycerin. Bettinger et al. [6] fanden im standardisierten Waschtest, dass nicht nur auf unbeeinflusster Haut, sondern auch auf gewaschener Haut der Wassergehalt der Hornschicht höher war, wenn eine glycerinhaltige Creme verwendet wurde als wenn die $\mathrm{O} / \mathrm{W}$ Grundlage allein zur Anwendung kam. Gloor und Schermer [7] konnten die hydratisierende Wirkung von Glycerin bestätigen und fanden im Gegensatz zu Harnstoff eine hochgradige Dosisabhängigkeit der Wirkung. Grunewald et al. [3] konnten die hydratisierende Wirkung im Langzeittest bestätigen. Bei repetitiven Waschungen und intermittierender Anwendung einer glycerinhaltigen O/W-Emulsion kann es zu einer hochgradigen Verminderung des durch die repetitiven Waschungen bedingten Austrocknungseffektes kommen. Fluhr et al. [8] konnten zeigen, dass der hydratisierende Effekt einer glycerinhaltigen Zubereitung im Vergleich zu deren Vehikel eine volle Woche nach Beendigung der Behandlung persistierte, wenn der Behandlung eine NLS-Irritation vorausgegangen war.

Die vorliegenden Untersuchungen lassen die Wirkung eines Lichterythems auf die Haut abschätzen. Die Lichtbehandlung führt nicht zu einer Austrocknung der Hornschicht, vielmehr bleibt an der unbehandelten Stelle in unseren Versuchen der Wassergehalt der Hornschicht nach der Lichtbestrahlung weitgehend konstant. Da après Präparate vor allem von Menschen mit trockener Haut (vorwiegend Atopiker) verwendet werden und da die Sonnenexposition vielfach im Zusammenhang mit ei- 
nem Bad erfolgt, wäre für derartige Zubereitungen eine hydratisierende Wirkung erwünscht.

Die vorliegenden Untersuchungen zeigen, dass die Prüfzubereitung mit Dexpanthenol und Glycerin die Hornschichtfeuchtigkeit beeinflusst. Dabei bestehen eindeutige Vorteile gegenüber einem Konkurrenzpräparat ohne Glycerin und Dexpanthenol. Es zeigt sich auch, dass sich die Wirkungen von Dexpanthenol und Glycerin addieren und dass die Kombination beider Wirkstoffe signifikante Vorteile gegenüber dem Vehikel erbringt. Die Kombination erscheint also als sinnvoll, da Glycerin in hoher Konzentration eine klebende Wirkung zeigt. Eine ähnliche additive Wirkung besteht bei Glycerin und Harnstoff [7]. Im Gegensatz dazu erwies sich die Kombination von Harnstoff und Ammoniumlactat nicht als effektiver als die von Harnstoff allein [9]. Die additive Wirkung von zwei Moisturizern ist dementsprechend keineswegs vorauszusetzen. Damit ist das wesentlichste Ergebnis der vorliegenden Untersuchungen, dass sich die Kombination von Glycerin und Dexpanthenol als effektiv erweist.

\section{Literatur}

${ }^{1}$ Gehring W, Gloor M. Effect of topically applied dexpanthenol on epidermal barrier function and stratum corneum hydration - Results of a human in vivo study. Arzneim Forsch 2000; 50: 659-663

${ }^{2}$ Gehring W, Gloor M. Der Effekt von Dexpanthenol bei experimentell geschädigter Haut. Z Hautkr 2001; 76: 212-218

${ }^{3}$ Grunewald AM, Gloor M, Gehring W, Kleesz P. Barrier creams - commercially available barrier creams versus urea and glycerol containing oil in water emulsions. Dermatosen 1995; 43: 69-74

${ }^{4}$ Gloor M, Gabard B, Fluhr JW, Lehmacher W. Action of an aluminium chlorohydrate and glycerol containing skin protection cream in experimental skin irritation produced by sodium laurylsulfate and solvents. Dermatol Beruf Umwelt 2001; 49: 67 - 70

${ }^{5}$ Berardesca E. EEMCO guidance for the assessment of stratum corneum hydratation: Electrical methods. Skin Res Technol 1997; 3: $126-132$

${ }^{6}$ Bettinger J, Gloor M, Gehring W. Influence of a pretreatment with emulsions on the dehydration of the skin by surfactants. Int J Cosm Sci 1994; 16: $53-60$

${ }^{7}$ Gloor M, Schermer S, Gehring W. Ist die Kombination von Harnstoff und Glycerin in Externagrundlagen sinnvoll? Z Hautkr 1997; 72: 509-514

${ }^{8}$ Fluhr JW, Gloor M, Lehmann L, Lazzerini S, Distante F, Berardesca E. Glycerol accelerates recovery of barrier function in vivo. Acta Derm Venereol (Stockh) 1999; 79: 418-421

${ }^{9}$ Gloor M, Fluhr J, Lehmann L, Gehring W. Do urea/ammonium lactate combinations achieve better skin protection and hydration than either component alone? Skin Pharmacol Appl Skin Physiol 2002; 15: 35-43

\section{Buchbesprechung}

\section{Farbatlas der Dermatoskopie}

Stolz, W., O. Braun-Falco, P. Bilek, W. H. C. Burgdorf, M. Landthaler

2. neubearb. u. erw. Aufl., 238 Seiten, 604 Abb. Blackwell, Berlin 2002, Geb. 94,95, ISBN 3-89412-357-5

In den letzten 20 Jahren hat die Auflichtmikroskopie und speziell die Dermatoskopie eine zunehmende Bedeutung für die klinische Differenzialdiagnose pigmentierter Hautveränderungen erfahren. Dabei gehört die schnelle und sichere Erkennung von malignen Melanomen zur wichtigsten Zielstellung der Dermatoskopie, bietet doch nur die exakte Früherkennung und damit die rechtzeitige Entfernung von Melanomen mit geringer Tumordicke die Chance für eine Heilung. Die Autoren geben mit ihrem „Farbatlas der Dermatoskopie“ gerade Ärzten, die in der Ausbildung oft wenig Gelegenheit erhalten, ihre Sicherheit in der klinischen Diagnostik von malignen Melanomen zu steigern, ein Instrumentarium von unschätzbarem Wert an die Hand. Die sorgfältig ausgewählten Farbfotos sind von brillanter Qualität, wobei das bereits erfolgreiche didaktische Konzept der Gegenüberstellung von makro- und mikroskopischen Aufnahmen besonders erwähnt werden soll. Dies erleichtert insbesondere dem weniger geübten Betrachter, die nötige Sicherheit bei der Differenzialdiagnose von Hautveränderungen zu erlangen. Das Bildmaterial wurde stark erweitert, wobei vor allem die neuesten Erkenntnisse zu pathologischen Veränderungen der Gesichtshaut berücksichtigt und eingearbeitet wurden. Dabei beschreibt der Text zu den insgesamt über 300 klinischen und dermatoskopischen Abbildungen nicht nur die relevanten Diagnosekriterien, sondern vermittelt dem Leser auch ein Basiswissen, auf das bei der täglichen Differenzialdiagnose aufgebaut werden kann.

Die exakte, konsequente und übersichtliche Gliederung des Buches in 13 Kapitel ermöglicht das schnelle und sichere Auffinden der gesuchten Abbildungen. Von den insgesamt ausgezeichnet recherchierten Kapiteln verdient das Kapitel 11 besonderes Augenmerk, wird doch dort ein integriertes Konzept für die digitale und computergestützte Dermatoskopie vorgestellt. Damit wird nicht nur einem Trend der „modernen Medien“ Rechnung getragen, sondern es werden auch die Grundlagen für das Anlegen von Datenbanken, Dokumentationen und nicht zuletzt für die Videound Teledermatologie berücksichtigt.

Das Autorenteam aus international anerkannten Kapazitäten bürgt dafür, dass der „Farbatlas der Dermatoskopie“ als eines der gelungensten Bücher der dermatologischen Literatur des Jahres 2002 bezeichnet werden kann und mit Sicherheit an den Erfolg der ersten Auflage anknüpfen wird.

Es verdient deshalb nicht nur jedem Dermatologen empfohlen zu werden, sondern sollte auch in keiner Praxis eines Internisten oder Hausarztes fehlen. Dies sollte dazu beitragen, getreu dem Motto des Autorenteams, die durch das Goethezitat „Man sieht nur, was man weiß“ inspiriert wurden, die Dermatoskopie zu entwickeln, den Blick für Veränderungen der Haut zu schärfen.

Uta-Christina Hipler, Jena 